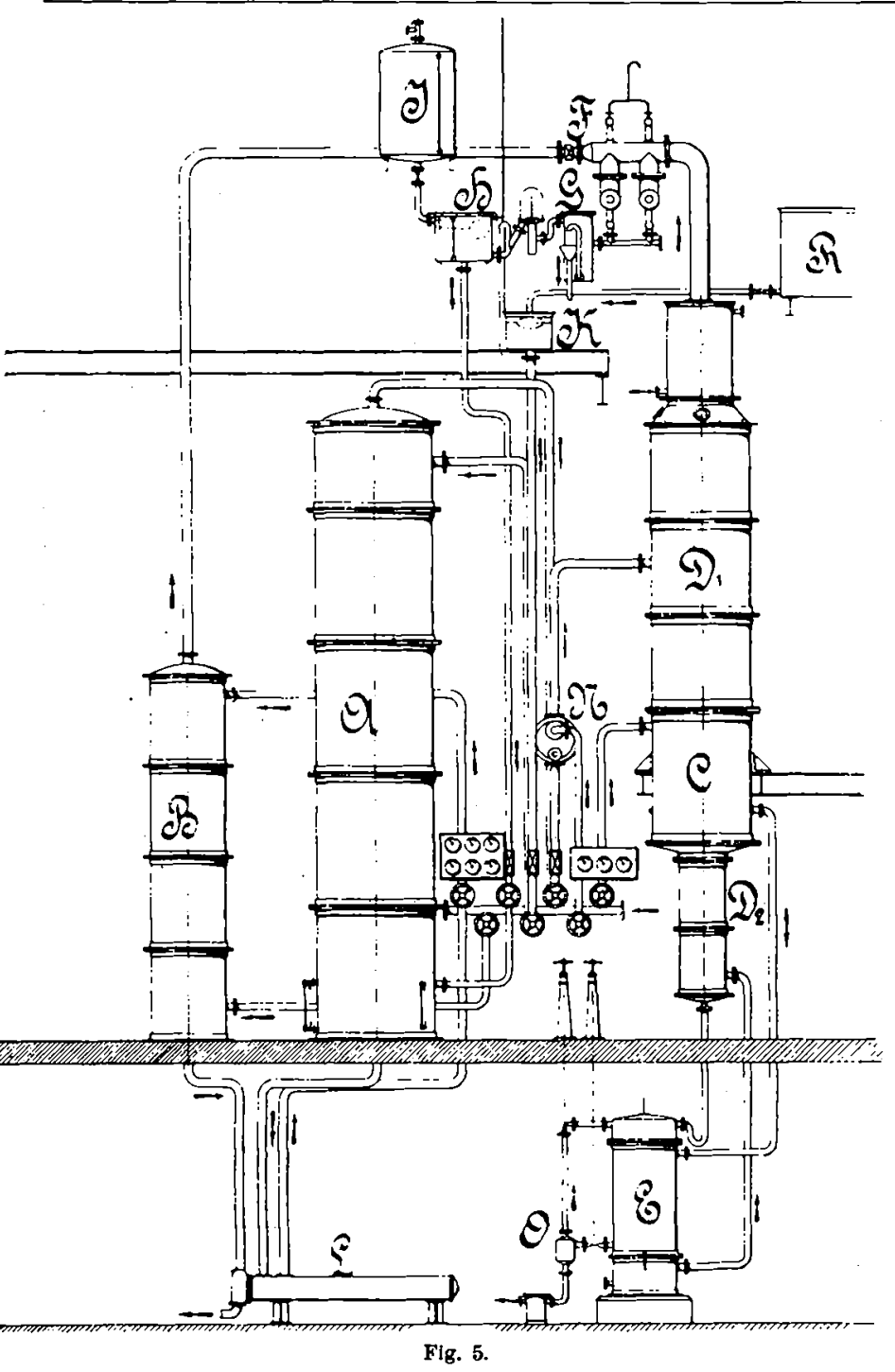

Die entbenzolierte Lauge gibt ihre Flüssigkeitswärme im Wärmeaustauscher $L$ ab und läuft gekühlt weg.

Die Anlage verarbeitet im Dauerbetrieb stündlich $8 \mathrm{cbm}$ Lauge, und zu ihrer Bedienung genügt im Notfall ein Mann. (Schlus folgt.)

\section{Brand feuergefährlicher Flüssigkeiten durch Blitzschlag und Verhütungsmaßregeln.}

Von Dr. Fritz Bennigson, Berlin-IVilmersdorf.

(Eingeg. 7./8. 1915.)

Ein gewaltiger Spiritusbrand in Königsberg in den ostelbischen Spritwerken an der Fuchsberger Allee hat über eine Woche hindurch die Mannschaften der städtischen Feuerwehr unter Oberleitung des Branddirektors Mat thes auf dieser Brandstätte festgehalten. Um das in den Spiritus tanks in der ersten Zeit weithin sichtbare, dann nach und nach schwächer werdende Feuer zu löschen, hat es fortgesetzter Bemühungen bedurft, denn von einer eigentlichen Löscharbeit bei diesem rauchlosen Dauerbrande konnte beinahe nicht gesprochen werden, da es sich in der Hauptsache um die unausgesetzte Verdünnung und Abkühlung der in dem großen Spiritustank enthaltenen Flüssigkeit gehandelt hat, die zum großen Teil in die auf dem Nachbarterrain an gelegten Gräben abgeleitet werden mußte. Auf diese Weise sank die Temperatur und damit die Entwicklung der entflammbaren Dämpfe, bis schließlich auch die letzten über dem Tank noch hin und her huschenden Flämmchen erloschen, und damit der 7. Tage dauernde Brand sein Ende fand.

Nach Aussage einiger im Hofe der Anlage anwesenden Personen scheint es erwiesen zu sein, daß ein Blitzstrahl das Dach eines Tanks mit einer Million Liter Spiritus darin getroffen hat. Eine Minute später sei eine mächtige, blaugelbe Flammo haushoch empor geschossen. Die Haupt- gefabr bestand nun darin, daß die Eisenwände der brennenden Tanks mit ihren mit Pappo belegten Dachkonstruktionen bersten konnten; in diesern Falle wäre die ungeheure Spiritusmenge trotz der die Tanks schützenden Frdumwallungen übergelaufen und ein Unglück von vielleicht unabsehbaren Folgen, da auch in der Anlage verschiedene kleine Tanks mit Benzinvorräten aufgestellt sind, entstanden. Um dieser Katastrophe vorzubeugen, ließ Herr Branddirektor Matthes die sämtlichen Rohre der Automobil- und Dampfspritze sowie cine Anzahl Handdruck spritzenrohre hochführen und unausgesetzt Wasser geben, um die Tanks, in denen der Spiritus unter deutlich vernehmbarem Poltern brocielte, zu kühlen. Die Flammen der brennenden Spiritusgase riefen nur während der Dunkelheit gegen den Himmel eine rote Glut hervor, während am Tage eine Rauchentwicklung nicht sichtbar war, so daß die mehrere Zentimeter dicken Wasserstrahlen der Dampfspritze anscheinend wirkungslos in das Feuer hineinzuprasseln schicnen. Der Schaden, welcher hier angerichtet ist, läßt sich mit Bestimmtheit zur Stunde noch nicht angeben, dürfte aber nach Schätzung von Sachverständigen mindestens eine halbe Million Mark betragen. Beteiligt sind daran 12 Versicherungsgesellschaften, die ostelbischen Spritwerke und in ganz erheblichem Maße auch der Steuerfiskus, dem für jeden durch den Brand vernichteten Liter Spiritus die Steuer ven $0,25 \mathrm{M}$ entgeht. Daneben ist der Materialschaden, den der Brand hervorgerufen hat, nicht unbedeutend, da auch die Nachbaranlagen Risse und Sprünge erlitten haben, und das umgebende Mauerwerk beschädigt ist.

Daß eine wirksame Verbütung solcher Katastrophen, wie wir sie hier wieder einmal erlebt haben, durch die bisherigen polizeilichen und berufsgenossenschaftlichen Vorschriften übèr die Aufbewahrung feuergefährlicher Flüssigkeiten nicht erreicht werden kann, ist wieder einmal bewiesen. Denn gegen Blitzgefahr und die Gefahren, die durch Selbstentzündung und durch elektrische Erregungen entstehen, sind eben dic bisherigen Bestimmungen unvollständig und veraltet, und es ist schließlich einerlei, ob eine weite oder eine enge Schutzzone verlangt wird, große oder kleine Mengen in der Nähe menschlicher Arbeits- und Verkehrsstätten zugelassen werden, brennen und explodieren kann es, solange eine Zündung möglich ist, in jedem Falle, und nur die Größe der Katastrophe ist ev. verschieden. Unvergeßlich ist wohl allen, die für diese Fragen wirkliches Interesse haben, der große Brand im Hafen von Antwerpen, bei welchem die Explosion $25 \mathrm{~km}$ gehört wurde; der Schaden ist auf ca. 7 Mill. Mark geschätzt, und ca. 25 Menschen sind getötet oder verunglückt. Ein schweres Unrecht wïrde es also sein, die Ansammlungsstätten großer Mengen feuergefährlicher Flüssigkeiten als harmlos hinzustellen, während in Wirklichkeit bei der jetzigen Aufbewahrungsart die schwersten Gefahren damit verbunden sind. Die Mittel, die die moderne Technik zur absoluten Sicherung von Vorräten feuergefährlicher Flüssigkeiten gegen Brandund Explosionsgefahren, auch bei Blitzschlag liefert, dürften daher von besonderem Interesse sein.

Geradezu hervorragende Leistungen auf diesem Gebiete, die ja auch bei allen Fachkreisen Anerkennung gefunden haben, sind niedergelegt in den Patenten der Herren $M$ a $r$ tin i und $H$ ü ne ke. Nicht nur für alle gewerblichen und privaten Bedürfnisse, sondern auch für die verschiedenen Industrien und die großen Umschlagläger in den Häfen sind hier die sämtlichen Vorbeugungsmaßnahmen wohl ausnahmslos zu finden. Eine besonders interessante Anlage nach diesem Verfahren hat ja auch kürzlich der Magistrat der Stadt Berlin inmitten der Reichshauptstadt dadurch geschaffen, dals er im neuerbauten Osthafen eine Million Liter der verschiedenartigsten feuergefährlichen Flüssigkeiten eingelagert hat in ca. 60 Einzeltanks zur Vermietung an die verschiedenen Verbraucher. Es ist infolgedessen wohl dringend zu wünschen, daß diese Sicherheitsmaßnahmen, die inzwischen auch bereits über die ganzen Kulturstaaten Verbreitung gefunden haben, besonders in den Festungen, wo feuergefährliche Flüssigkeiten vorrätig gehalten werden müssen, zur Sicherstellung derselben für wichtige Verbrauchsfälle und ebenso zum Schutze für Leben und Gut Anwendung finden.
[A. 96.] 